\title{
Les éclampsies en centre hospitalier universitaire en Côte d'Ivoire: prise en charge, évolution et facteurs pronostics
}

\author{
[Eclampsia at a teaching hospital in Ivory Coast: management, outcome and
} prognostic factors]

Yapo Brouh PhD, ${ }^{*}$ Patrick Gimel Ndjeundo MD, ${ }^{*}$ Yavo Denis Tetchi MD,${ }^{*}$ Antoine Akpo Amonkou PhD,$\dagger$ Yaïch Pete MD, ${ }^{*}$ Yves Yapobi PhD $\dagger$

Objectif : Analyser les aspects thérapeutiques et évolutifs de l'éclampsie en milieu de réanimation en Côte d'Ivoire.

Méthode : Étude rétrospective réalisée au sein des services de réanimation des Centres Hospitaliers Universitaires $(\mathrm{CHU})$ de Cocody et de Yopougon de 200I à 2006. Les patientes admises en réanimation pour éclampsie ont été incluses dans l'étude. Les aspects thérapeutique et évolutif (état clinique à l'admission, médicaments utilisés, mode d'accouchement, type d'anesthésie, létalité, séquelles, l'évolution du tableau clinique) ont été étudiés. Les facteurs prédictifs de décès étaient recherchés par le calcul du risque relatif assorti de son intervalle de confiance. Le Chi carré de Mantel Haenszel a été utilisé avec une erreur $\alpha=0,05$.

Résultats : L'étude a porté sur 313 patientes dont l'âge moyen était de $22,7 \pm 6,1$ ans. Le diazépam était l'anticonvulsivant le plus utilisé $(50,5 \%$ des cas) et la dihydralazine l'hypertenseur le plus fréquemment employé $(50,2 \%)$. L'accouchement a été réalisé par césarienne dans $58,5 \%$ des cas et par voie vaginale dans $41,5 \%$ des cas. Pour la césarienne, l'anesthésie générale avec intubation a été réalisée dans $79 \%$ des cas et la rachianesthésie utilisant la bupivacaïne 0,5\% (dose: 10 à $12,5 \mathrm{mg}$ ) dans $21 \%$ des cas. Le traitement n'a pas été régulièrement suivi dans $31,3 \%$ des cas. La mortalité maternelle s'élevait à 16 $\%$ et la mortalité périnatale à $16,1 \%$. La provenance hors du $\mathrm{CHU}$, le délai d'admission $>12 \mathrm{~h}$, le score de Glasgow $\leq$ à 8 à l'admission, l'état de mal éclamptique et le suivi irrégulier du traitement étaient les facteurs prédictifs de mortalité.

Conclusion : La mortalité maternelle est encore élevée dans notre contexte. Une action énergique sur les facteurs de mauvais pronostics permettra de réduire la mortalité.
CAN J ANESTH 2008/55: 7 / pp. 423-428

Objective: To analyze the management and evolution of eclampsia in the intensive care units of Côte d'lvoire.

Methodology: Retrospective study undertaken in the intensive care units in the University Teaching Hospitals of Cocody and Yopougon from $200 \mathrm{I}$ to 2006. Patients admitted to the intensive care unit for eclampsia were included in this study. The management and evolution (clinical condition at admission, medications used, type of delivery, type of anesthesia, mortality, sequellae, clinical evolution) were studied. Factors predicting mortality were identified with the calculation of odds ratio and confidence interval. The Chi-square of Mantel Haenszel was used with an $\alpha$ error $=5 \%$.

Results: The study involved $3 / 3$ patients with a mean age of $22.7 \pm 6.1$ yr. Diazepam was the most frequently used anticonvulsant $(50.5 \%$ of cases), and dihydralazine was the preferred antihypertensive agent $(50.2 \%)$. Cesarean delivery occurred in $58.5 \%$ of cases and vaginal delivery in $41.5 \%$. General anesthesia with intubation was used in $79 \%$ of Cesarean deliveries and spinal anaesthesia with bupivacaine $0.5 \%$ (dose: 10 in $12.5 \mathrm{mg}$ ) in $21 \%$. Treatment was not followed regularly in $31.3 \%$ of cases. Maternal and perinatal mortality was $16 \%$ and $16.1 \%$, respectively. Risk factors for mortality were: admission from outside of a university hospital, admission delay $>12 \mathrm{hr}$, Glasgow score $\leq 8$ on admission, status eclampticus and poor compliance to therapy.

Conclusion: Maternal mortality is still high in our setting. Vigorous action on the factors associated with bad prognosis should yield a reduction in mortality rate.

Des services d'anesthésie-réanimation, CHU de Cocody, ${ }^{*}$ et CHU de Yopougon, $†$ Côte d'Ivoire. Adresser la correspondance à : Pr Brouh Yapo, CHU de Cocody, 22 BP 1771 Abidjan 22, Côte d'Ivoire. Courriel : brouh_yapo@yahoo.fr

Accepté pour publication le 29 octobre 2007.

Révision acceptée le 4 décembre 2007.

Version finale acceptée le 3 mars 2008.

Cet article est accompagné d'un éditorial. Veuillez consulter Can J Anesth 2008; 55: 397-402. 
$\mathrm{L}$

'ÉCLAMPSIE est une complication neurologique majeure de la toxémie gravidique. Elle est caractérisée par des convulsions survenant classiquement chez la jeune primipare au cours du troisième trimestre de la grossesse, avant, pendant ou après l'accouchement. ${ }^{1}$ Dans les pays développés, on note une incidence de 27-56/100 000 naissances contre 320-1 075/100 000 naissances dans les pays sous développés qui ont également un taux de mortalité élevé (6 à $35 \%$ ). ${ }^{2}$ Une meilleure surveillance des grossesses et une prise en charge optimale de ces patientes en milieu de réanimation pourraient réduire son incidence et améliorer le pronostic de l'éclampsie. Nous avons entrepris cette étude afin d'analyser les aspects thérapeutiques et évolutifs de la maladie et pour rechercher les facteurs qui influencent négativement la morbidité et la mortalité en milieu de réanimation en Côte d'Ivoire.

\section{Méthodes}

Il s'agissait d'une étude rétrospective sur dossiers qui avait eu lieu au sein des services de réanimation des Centres Hospitaliers et Universitaires (CHU) de Cocody et de Yopougon sur une période de cinq ans (de janvier 2001 à décembre 2006). Les patientes admises aux soins intensifs pour éclampsie et ayant bénéficié d'une prise en charge thérapeutique dans les services ont été incluses dans l'étude. Les patientes ayant des dossiers incomplets ou non retrouvés, chez qui le diagnostic d'éclampsie n'est pas confirmé, ou qui ont une autre cause pour expliquer la convulsion ont été exclues. Les paramètres portant sur les aspects clinique, thérapeutique et évolutif (âge, délai d'admission, provenance, terme de la grossesse, pression artérielle systolique non invasive à l'admission, existence de pré-éclampsie, période de survenue de la crise d'éclampsie, médicaments utilisés, mode d'accouchement, type d'anesthésie, pose de cathéter veineux central avec mesure de la pression veineuse centrale (PVC), intubation trachéale, ventilation mécanique, décès, séquelles, durée de séjour aux soins intensifs, l'évolution du tableau clinique) ont été étudiés. De même, les facteurs pronostics de décès ont été soulignés. Le délai d'admission était le temps écoulé entre la première crise convulsive et l'admission de la patiente aux soins intensifs. La recherche d'une association entre les facteurs pronostics et le décès maternel s'est faite par le calcul des risques relatifs assorti de son intervalle de confiance. La stabilité de l'association a été évaluée par le test de Chi carré de Mantel Haenszel avec une erreur $\alpha=0,05$.
TABLEAU I Traitement anticonvulsivant

\begin{tabular}{lll}
\hline Traitement anticonvulsivant & $\begin{array}{l}\text { Nombre de } \\
\text { patientes }\end{array}$ & Pourcentage \\
\hline Diazépam & 158 & 50,5 \\
Valproate de sodium & 33 & 10,5 \\
Phénobarbital & 7 & 2,2 \\
Diazépam + phénobarbital & 51 & 16,3 \\
Diazépam + valproate de sodium & 23 & 7,3 \\
Aucun & 41 & 13,1 \\
Total & 313 & 100 \\
\hline
\end{tabular}

\section{Résultats}

Durant la période, nous avons recensé 341 cas d'éclampsies dont 313 répondaient à nos critères d'inclusion. L'âge moyen des patientes était de [moyenne \pm écart-type (extrêmes)] 22,7 $\pm 6,1$ (14-44) ans. Le délai d'admission moyen était de 17,4 \pm 19,3 (1-192) h. Plus de la moitié (54\%) des patientes étaient admises au-delà de $12 \mathrm{~h}$. La majorité des patientes $(87,5 \%)$ provenait des services de gynéco-obstétrique des Centres Hospitaliers Universitaires (CHU) de Yopougon et de Cocody, et 12,5\% provenaient des formations sanitaires périphériques. La grossesse était au-delà de la 37 e semaine dans $61,4 \%$ des cas. La pression artérielle systolique non invasive à l'admission était de $164 \pm 28(60-280) \mathrm{mmHg}$. Elle était $\geq 160$ $\mathrm{mmHg}$ chez $64,6 \%$ des patientes. Les patientes ont présenté une pré-éclampsie suivie d'éclampsie dans $84 \%$ $(n=263)$ des cas. Les autres, soit $16 \%(n=50)$ ont présenté de façon inaugurale une crise d'éclampsie. La crise d'éclampsie survenait le plus souvent dans le prépartum $(60 \%)$ mais aussi dans le per-partum $(8,6 \%)$ et le post-partum $(31,4 \%)$. Le diazépam, seul ou en association, était le traitement anticonvulsivant le plus souvent entrepris (Tableau I). L'accouchement a été réalisé dans plus de la moitié des cas par césarienne $(58,5 \%)$ et par voie vaginale dans $41,5 \%$ des cas. En cas de césarienne, une anesthésie générale au thiopental avec intubation était réalisée le plus souvent ( $n=145 ; 79 \%$ des césariennes), la rachianesthésie utilisant la bupivacaïne 0,5\% (dose: 10 à 12,5 mg) étant choisie dans les $21 \%$ restants $(n=38)$. Dans $40,3 \%$ des cas $(n=126)$, les patientes ont bénéficié de la pose d'un cathéter veineux central et aucune n'a eu un cathéter de Swan Ganz. Une hypovolémie relative ( $\mathrm{PVC}<3 \mathrm{~cm} \mathrm{H}_{2} \mathrm{O}$ ) a été notée chez 95,2\% $(n=120)$ des patientes ayant eu une voie veineuse centrale. Cette hypovolémie a été corrigée par perfusion de cristalloïdes (sérum salé isotonique et Ringer's lactate) chez la plupart des patientes $(88,5 \%)$ sous une surveillance clinique rigoureuse et une évaluation 
TABLEAU II Traitement antihypertenseur

\begin{tabular}{lll}
\hline Antihypertenseur & Effectif & Pourcentage $(\%)$ \\
\hline Dihydralazine & 157 & 50,1 \\
Nicardipine & 79 & 25,3 \\
Nifédipine & 20 & 6,4 \\
Clonidine & 25 & 8,0 \\
A methyl dopa & 13 & 4,2 \\
Aucun & 19 & 6,1 \\
Total & 313 & 100 \\
\hline
\end{tabular}

régulière de la PVC. L'intubation trachéale a été réalisée chez $46,3 \%$ des patientes qui présentaient un coma et une détresse respiratoire avec encombrement trachéo-bronchique important. La ventilation mécanique s'est avérée nécessaire chez $23 \%$ des patientes intubées. Les antihypertenseurs utilisés ont été notés au Tableau II. Chez 52,4 \% des patientes, on a eu recours à un traitement diurétique après un remplissage vasculaire correct soit avec du furosémide seul $(49,5 \%)$ soit en association avec la dopamine à dose dopaminergique $(2,9 \%)$. Dans un peu plus d'un tiers des cas $(33,5 \%)$ on a transfusé du concentré érythrocytaire isogroupe isorhésus et du plasma frais congelé. La grande majorité des patientes $(72,9 \%)$ a bénéficié d'un traitement anticoagulant à base d'héparine de bas poids moléculaire $(72,2 \%)$. Les antalgiques (paracétamol et/ou tramadol) ont été utilisés dans (84,7\%) des cas, le kétoprofềne dans $2 \%$. Il est à noter que 13,3\% des patientes n'ont pas bénéficié d'un antalgique. Le traitement, pris en charge totalement par les parents, n'a pas été régulièrement suivi (impossibilité pour les parents d'honorer les médicaments et les bilans paracliniques certains jours) chez $31,3 \%$ des patients. L'évolution a été émaillée d'une mortalité de $16 \%$. Plus des trois quarts $(76,7 \%)$ des patientes sont sorties du service sans aucune séquelle. Les séquelles observées sont résumées au Tableau III. La durée moyenne de séjour aux soins intensifs était de 4,0 $\pm 3,7$ (1-33) jours. La plupart des patientes $(76,1 \%)$ ont eu une durée d'hospitalisation en réanimation comprise entre deux et six jours. La tension artérielle systolique s'est normalisée chez $72,5 \%$ des patientes $(<140 \mathrm{mmHg})$ dans les $72 \mathrm{~h}$ après le début du traitement. Chez la grande majorité des patientes $(78,3 \%)$, on a obtenu une amélioration du score de Glasgow après les 48 premières heures de traitement. La reprise d'une diurèse normale s'est faite dans $86,9 \%$ des cas, après trois jours de traitement. Le séjour était émaillé de plusieurs complications : infectieuses (15\%), mal éclamptique $(6,4 \%)$, insuffisances rénales aiguës $(6,4 \%)$, accidents vasculaires cérébraux hémorragiques $(5,1 \%)$, HELLP
TABLEAU III Répartition selon les séquelles observées à la sortie

\begin{tabular}{lll}
\hline Séquelles & Effectif & Pourcentage (\%) \\
\hline Aucune & 240 & 76,7 \\
Hémiparésie & 11 & 3,5 \\
Hémiplégie & 6 & 1,9 \\
Insuffisance rénale chronique & 4 & 1,3 \\
Rétinopathie hypertensive & 1 & 0,3 \\
Ascite réfractaire & 1 & 0,3 \\
Décès & 50 & 16,0 \\
TOTAL & 313 & 100 \\
\hline
\end{tabular}

syndrome $(3,9 \%)$. Les principaux facteurs de mauvais pronostics maternels sont résumés dans le Tableau IV. On a noté une mortalité foetale tardive dans 23 cas et une mortalité néonatale précoce dans 26 cas (16,1\%). Il s'agissait de 31 enfants nés par voie vaginale et de 18 enfants nés par césarienne.

\section{Discussion}

Notre étude montre un délai moyen d'admission aux soins intensifs de plus de $17 \mathrm{~h}$ soit plus d'une demijournée après la première crise. Cette tendance au retard d'admission se confirme dans plusieurs études menées en Afrique dont celle de $\operatorname{Beye}^{3}(18,5 \mathrm{~h})$ au Sénégal en 1999 et celle de Soro ${ }^{4}(12,6$ h) en 2000 au CHU de Yopougon.

\section{Au plan thérapeutique}

Le diazépam a été aussi l'anticonvulsivant le plus utilisé, comme dans l'étude de Soro. ${ }^{4}$ Mais il est important de rappeler ici la place du sulfate de magnésium dans le traitement anticonvulsivant notamment dans les pays anglo-saxons ${ }^{5}$ et de plus en plus en Ukraine, ${ }^{6}$ en Irlande ${ }^{7}$ et en Suède. ${ }^{8}$ Le sulfate de magnésium diminuerait le risque de survenue de récidive des crises convulsives avec moins de complications respiratoires. ${ }^{5}$ Mais l'utilisation du sulfate de magnésium pose le problème du choix des antihypertenseurs administrés. En effet il existe un risque de potentialisation de l'effet des inhibiteurs calciques lors de leur association entraînant alors une baisse trop importante de la pression artérielle diastolique toujours préjudiciable au fotus, ${ }^{9}$ de plus il est démontré actuellement que le sulfate de magnésium est un puissant agent tocolytique ${ }^{10}$ qui retarde le déclenchement spontané du travail alors que l'extraction foeto-placentaire constitue le véritable traitement étiologique.

Le monitorage de la volémie dans l'éclampsie s'avère indispensable pour mieux contrôler les troubles hémodynamiques pouvant aggraver les nombreuses com- 
TABLEAU IV Facteurs pronostics identifiés

\begin{tabular}{lllllll}
\hline Facteurs pronostics & Nombre & Survie & Décès & RR & IC 95 \% & $P$ \\
\hline Provenance hohrs du CHU & 39 & 27 & 12 & 2,76 & $(1,20-6,27)$ & 0,0071 \\
Gestité > & 26 & 18 & 8 & 0,39 & $(0,15-1,4)$ & 0,031 \\
Délai d'admission $>12 \mathrm{~h}$ & 150 & 117 & 33 & 2,42 & $(1,23-4,79)$ & 0,0053 \\
Score de Glasgow $\leq 8$ & 99 & 59 & 40 & 13,83 & $(6,21-31,55)$ & 0,000001 \\
État de mal éclamptique & 22 & 7 & 15 & 15,67 & $(5,50-46,06)$ & 0,000001 \\
Suivi du traitement irrégulier & 98 & 69 & 29 & 3,88 & $(1,99-7,61)$ & 0,000009 \\
\hline
\end{tabular}

$\mathrm{RR}=$ risque relatif $; \mathrm{IC}=$ intervalle de confiance $; \mathrm{CHU}=$ centre hospitalier universitaire.

plications déjà pré-existantes. ${ }^{\mathrm{A}}$ On a posé un cathéter veineux central chez $40,3 \%$ des patientes de notre série. Notre étude a permis de constater que 95,2 \% des patientes ayant une voie veineuse centrale avaient une PVC négative, confirmant ainsi les écrits sur la physiopathologie de l'éclampsie, quant à l'existence d'une hypovolémie relative. ${ }^{\mathrm{A}, 11}$ La PVC doit être monitorée chez les éclamptiques afin d'éviter les risques inhérents aux variations extrêmes de la PVC : œedème aigu du poumon si elle est élevée, ischémie viscérale et œedème cérébral si elle est basse. Le remplissage vasculaire a été réalisé chez $88,5 \%$ des patientes avec des cristalloïdes sous surveillance des constantes hémodynamiques et de la PVC. Dans l'étude de Gilbert et coll. ${ }^{12}$ menée conjointement en Californie (USA) et à Cape Town (Afrique du Sud) en 1997, on remarque que le remplissage vasculaire a été pratiqué chez toutes les patientes avant même la pose d'un cathéter veineux central. De plus l'expérience montre que la correction simple et efficace de l'hypovolémie suffit généralement à ramener les chiffres tensionnels à des niveaux à peu près acceptables. ${ }^{12}$ En outre, ce remplissage vasculaire précédant un traitement antihypertenseur éventuel prévient une chute tensionnelle trop brutale qui pourrait être responsable d'ischémies graves essentiellement au niveau cérébral et rénal chez la mère mais également chez le foetus. ${ }^{4,13}$ Certains auteurs se gardent donc de mettre systématiquement un traitement antihypertenseur et préconisent uniquement un bon remplissage vasculaire sous une surveillance rigoureuse de la PVC avec un traitement symptomatique, qui peut comprendre des antalgiques. ${ }^{\mathrm{A}}$

La plupart des patientes ont reçu un traitement antihypertenseur par voie intraveineuse, essentiellement avec de la dihydralazine $(50,1 \%)$ ou de la nicardipine $(25,3 \%)$. Il en est de même dans l'étude de Soro et coll.: $65 \%$ de dihydralazine et $7,5 \%$ de nicardipine. Le principe était la surveillance de l'évolution des chif-

A Palot $M$, Kessler P, Visseaux H, Botmans C. Toxémie gravidique. SFAR Conférence d'Actualisation, $38^{\text {ème }}$ Congrès National d'Anesthésie et de Réanimation. Paris: Elsevier; 1997: 633-52. fres tensionnels et leur réduction progressive. Nous remarquons dans notre étude que 19 patientes $(6,1$ $\%)$ ont eu une normalisation des chiffres tensionnels, après évacuation utérine et correction de l'hypovolémie relative sous contrôle de la PVC et sans aucun antihypertenseur.

Nous avons eu recours au diurétique dans $52,4 \%$ des cas après un remplissage vasculaire correct. Notre taux est inférieur à celui de Gilbert et coll. ${ }^{12}$ qui était de $71 \%$. Par ailleurs il a été noté une reprise de la diurèse dans les $72 \mathrm{~h}$ après le début des diurétiques chez $85 \%$ des patients présentant une oligurie. L'oligurie chez l'éclamptique est due à une insuffisance rénale fonctionnelle qui est réversible. ${ }^{1,13}$ Seule l'ischémie rénale prolongée due à l'hypovolémie peut entrainer une insuffisance rénale organique. ${ }^{13}$

Dans notre série, $23 \%$ des patientes ont bénéficié d'une assistance ventilatoire. Rugarn et coll. ${ }^{8}$ en Suède et Beye et coll. ${ }^{3}$ au Sénégal trouvaient respectivement un taux de $35 \%$ et de $71 \%$. La ventilation mécanique était réalisée après libération des voies aériennes chez des patientes ayant eu plusieurs épisodes de crises convulsives avec développement d'un œdème cérébral et d'une hypertension intracrânienne.

La majorité des patientes ont bénéficié d'un traitement antalgique, le plus souvent à base de paracétamol $(57,5 \%)$ en perfusion. Cela s'avère justifié dans la mesure où la majorité d'entre elles ont eu un accouchement par césarienne et souffrait d'une douleur qui pouvait entretenir l'hypertension artérielle. ${ }^{11}$

L'irrégularité dans le suivi du traitement dans 31,3\% des cas se justifie par l'épuisement financier des parents, difficultés financières qui survenaient principalement après quatre à cinq jours d'hospitalisation.

\section{Au plan épolutif}

La mortalité maternelle dans notre série était de $16 \%$. Ce taux de mortalité a tendance à régresser en Afrique, même s'il reste élevé comparativement aux pays développés; en effet Bouaggad et coll. ${ }^{14}$ retrouvaient 23,3 \% au CHU Ibn Rochd de Casablanca en 1995, Beye et coll. ${ }^{3}$ ont noté un taux de $35 \%$ à l'hôpital 
Aristide Le Dantec de Dakar en 1999. Tous ces chiffres étaient supérieurs à ceux que nous retrouvons aujourd'hui. ${ }^{15,16}$ Ailleurs dans les pays développés, ce taux est variable selon les séries : $0 \%$ pour Jenkins et coll. ${ }^{15}$ à Birmingham); $10 \%$ pour la série de Tang et coll. ${ }^{18}$ en Chine.

Toutes les complications de l'éclampsie ont été observées, et étaient associées entre elles dans des proportions diverses. Dans l'étude de Pambou et coll. ${ }^{2}$ c'est l'état de mal éclamptique qui venait en tête avec $47,6 \%$ suivi des accidents vasculaires cérébraux $(7,1 \%)$ et de l'œedème aigu du poumon $(4,8 \%)$. Miguil et coll. ${ }^{\mathrm{B}}$ trouvaient $29 \%$ de complications neurologiques, $24 \%$ d'insuffisance rénale aigue et $9 \%$ de HELLP syndrome à Casablanca en 2001.

Nous avons retrouvé une durée d'hospitalisation en réanimation dans la plupart des cas de deux à sept jours avec une durée moyenne de quatre jours ; Jenkins et coll. ${ }^{15}$ à Birmingham trouvaient une durée de séjour allant de un à six jours. Ben Letaifa et coll. ${ }^{19}$ en Tunisie et Moussaoui et coll. ${ }^{\mathrm{C}}$ au Maroc trouvaient respectivement une durée moyenne de séjour de six et 7,8 jours.

Les facteurs de mauvais pronostics retrouvés dans notre série (Tableau IV) étaient la provenance en dehors des CHU, le délai d'admission >12 h, l'altération grave de l'état de conscience avec un score de Glasgow $\leq 8$, l'état de mal éclamptique et le traitement irrégulier. La gestité élevée quant à elle, était un facteur de bon pronostic comme le souligne la plupart des études sur l'éclampsie.

\section{Conclusion}

Au terme de cette étude, nous avons observé que l'éclampsie aux soins intensifs est une pathologie dont le pronostic demeure grave dans notre contexte. Certains facteurs ont été identifiés comme facteurs de mauvais pronostics. Une action préventive et énergique sur ces facteurs de mauvais pronostics permettra sans doute de réduire la mortalité maternelle.

\section{Références}

1 Saissy JM, Vachon F. La toxémie gravidique. Dans: Saissy JM (Ed.). Précis de Réanimation Tropicale. Paris : Arnette; 1998: 959-72.

B Mignil M, Salmi S, Moubaoni M, Youssoufi EI. Aspects épidémiologiques et pronostiques de l'éclampsie. Cah d'anesth (Paris) 2003; 51:177-80.

C Moussaoui A, El Himdy N, Benyacob A, El ghadbane H, Tachinante R, Tazi AS. Morbidité et mortalité de l'éclampsie au CHU Avicienne (Rabat, Maroc). Cah d'anesth (Paris) 2002; 50: 319-23.
2 Pambou O, Ekoundzola JR, Malanda JP, Buambos S. Prise en charge et pronostic de l'éclampsie au C.H.U. de Brazzaville : à propos d'une étude rétrospective de 100 cas. Méd Afr Noire 1999; 46: 508-12.

3 Beye MD, Diouf E, Kane $O$, et al. Prise en charge de l'éclampsie grave en réanimation en milieu tropical africain. A propos de 28 cas. Ann Fr Anesth Réanim 2003; 22: 25-9

4 Soro L, Koname KE, Brouh $\Upsilon$, Yeo T, Aye YD. Analyse de la prise en charge des éclampsies graves en réanimation au CHU de Yopougon. Rev Inter des Sciences Médicales 2000; 2: 29-34.

5 Duley L, Henderson-Smart D. Magnesium sulphate versus phenytoin for eclampsia. Database Syst Rev 2000; (2): CD000128.

6 Fujikawa DG. Anticonvulsants for eclampsia. Lancet 1995; 346: 501-2.

7 Murray D, O'Riordan M, Geary M, Phillips R, Clarke T, McKenna P. The HELLP syndrome: maternal and perinatal outcome. Ir Med J 2001; 94: 16-8.

8 Rugarn O, Carling Meon S, Berg G. Eclampsia at a tertiary hospital 1973-99. Acta Obstet Gynecol Scand 2004; 83: 240-5.

9 Waisman GD, Mayorga LM, Camera MI, Vignolo CA, Martinotti $A$. Magnesium plus nifedipine: potentiation of hypotensive effect in preeclampsia? Am J Obstet Gynecol 1988; 159: 308-9.

10 Tsatsaris V, Carbonne B, Cabrol D. Les nouveaux tocolytiques. Dans: Mises à jour en Gynécologie Obstétrique. Collège National des Gynécologues et Obstétriciens Français. Paris : Éditions Vigot; 2001: 183-207.

11 Edouard D. Pré éclampsie/éclampsie. Encycl Méd Chir (Paris) 2003; 36-980-A-10.

12 Gilbert WM, Towner DR, Field NT, Anthony J. The safety and utility of pulmonary artery catheterization in severe preeclampsia and eclampsia. Am J Obstet Gynecol 2000; 182: 1397-403.

13 Fournie A, Bernadet P, Desprats R. Syndromes vasculorénaux de la grossesse. Encycl Méd Chir (Paris). 1995; 5036-A-10: 20

14 Bouaggad A, Laraki M, Bouderka MA, et al. Maternal prognostic factors in severe eclampsia (French). Rev Fr Gynecol Obstet 1995; 90: 205-7.

15 Jenkins SM, Head BB, Hauth JC. Severe preeclampsia at $<25$ weeks of gestation: maternal and neonatal outcomes. Am J Obstet Gynecol 2002; 186: 790-5.

16 Cisse CT, Faye Dieme ME, Ngabo D, Mbaye M, Diagne $P M$, Morean JC. Indications thérapeutiques et pronostic de l'éclampsie au CHU de Dakar. J Gynecol Obstet Biol Reprod (Paris) 2003; $32(3$ Pt 1): 239-45. 
17 Mayi-Tsonga S, Akouo L, Ngou-Mve-Ngou JP, Meye JF. Facteurs de risque de l'éclampsie à Libreville (Gabon): étude cas-témoins. Sante 2006; 16: 197-200.

18 Tang LC, Kwok AC, Wong AY, Lee YY, Sun KO, So $A P$. Critical care in obstetrical patients: an eight-year review. Chin Med J (Engl) 1997; 110: 936-41.

19 Ben Letaifa D, Daouas N, Ben Jazia K, Slama A, Jegham $H$. Maternal emergencies requiring controlled ventilation: epidemiology and prognosis (French). J Gynecol Obstet Biol Reprod (Paris) 2002; 31: 256-60.

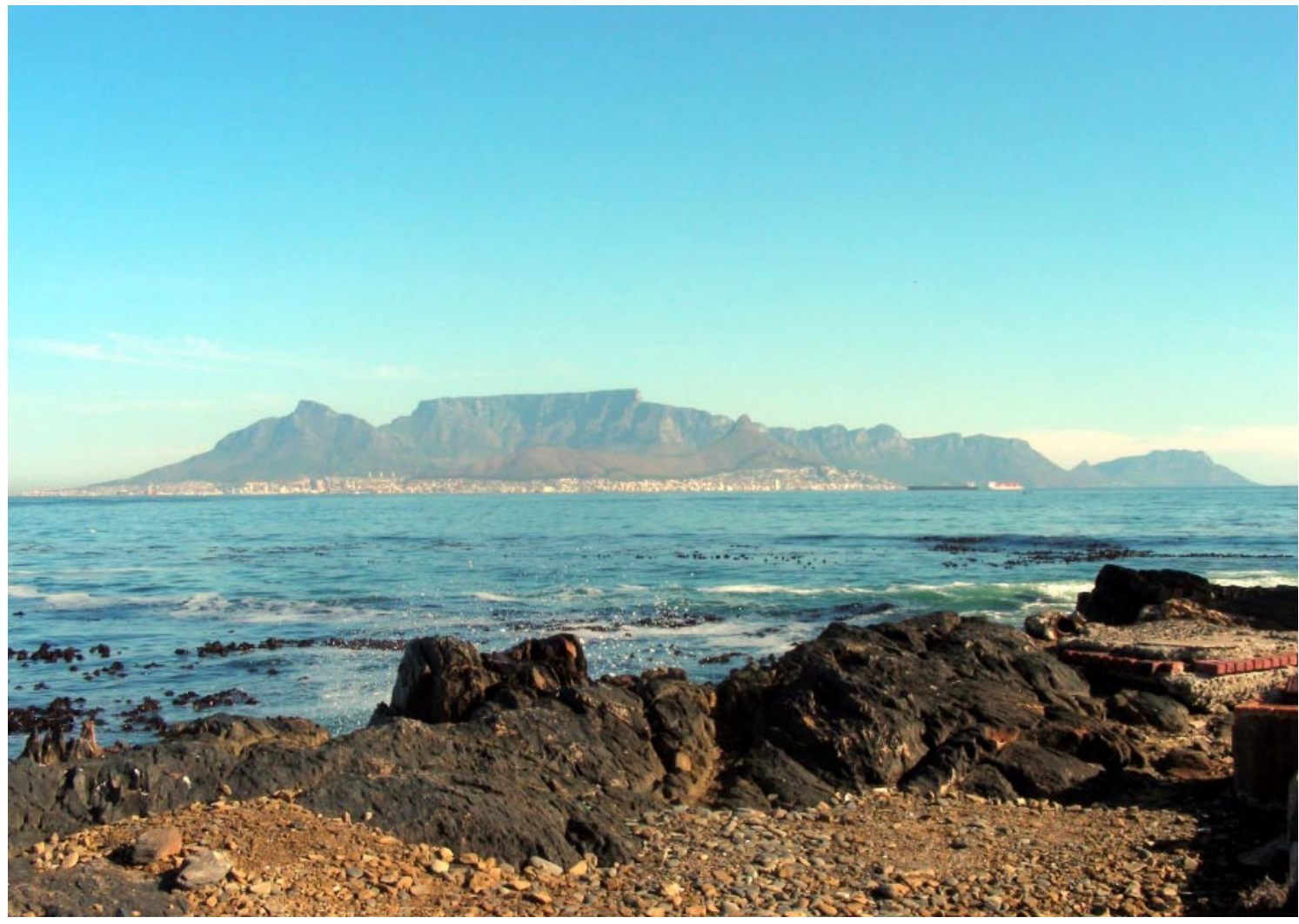

Robben Island - South Africa 